\title{
Safety of low-volume PEG-asc bowel cleansing preparation for colonoscopy: identifying patients at risk for hypokalemia in a prospective cohort study
}

\section{(ㄷ)(1) $(-)$}

Authors

Ankie Reumkens ${ }^{1,2,3}$, Christine Minke Bakker ${ }^{1,2}$, Sebastiaan J.W. van Kraaij ${ }^{2}$, Bjorn Winkens ${ }^{4,5}$, Maarten T. Raijmakers $^{6}$, Annick B. van Nunen', Cees Th.B.M. van Deursen ${ }^{1,2}$, Ad A.M. Masclee ${ }^{2,3}$

Institutions

1 Department of Internal Medicine and Gastroenterology, Zuyderland Medical Center, Sittard-Heerlen, the Netherlands

2 Division of Gastroenterology and Hepatology, Department of Internal Medicine, Maastricht University Medical Center, Maastricht, the Netherlands

3 NUTRIM, School for Nutrition, Toxicology and Metabolism, Maastricht University Medical Center, Maastricht, the Netherlands

4 Department of Methodology and Statistics, Maastricht University Medical Center, Maastricht, the Netherlands

5 CAPHRI, Care and Public Health Research Institute, Maastricht University Medical Center, Maastricht, the Netherlands

6 Department of Clinical Chemistry and Haematology, Zuyderland Medical Center, Sittard-Geleen, the Netherlands

submitted 15.12 .2020

accepted after revision 1.3.2021

Bibliography

Endosc Int Open 2021; 09: E1198-E1204

DOI 10.1055/a-1478-3361

ISSN 2364-3722

(c) 2021. The Author(s).

This is an open access article published by Thieme under the terms of the Creative Commons Attribution-NonDerivative-NonCommercial License, permitting copying and reproduction so long as the original work is given appropriate credit. Contents may not be used for commercial purposes, or adapted, remixed, transformed or built upon. (https://creativecommons.org/licenses/by-nc-nd/4.0/)

Georg Thieme Verlag KG, Rüdigerstraße 14,

70469 Stuttgart, Germany

Corresponding author Ankie Reumkens, MD, Division of Gastroenterology and Hepatology, Department of Internal Medicine, Maastricht University Medical Center, Postbox 5800, 6202 AZ, Maastricht, The Netherlands
Fax: +31-43-3875006

a.reumkens@mumc.nl

\section{ABSTRACT}

Background and study aims The aim of bowel cleansing preparation should be high-quality results and conformance with safety standards. Previously, we reported that hypokalemia occurred in $23.6 \%$ of patients after bowel preparation in a high-risk population on diuretics or hospitalized and referred for colonoscopy. Here we report on a prospective study in a non-selected colonoscopy cohort to identify patients at risk of developing hypokalemia before and after bowel cleansing with low-volume polyethylene glycol with ascorbic acid (PEG-asc).

Patients and methods From January 1 to July 31, 2016, we included all patients undergoing colonoscopy in our institution. Prevalences of hypokalemia before and after PEGasc bowel cleansing for colonoscopy were calculated and risk factors for developing hypokalemia after PEG-asc bowel cleansing were identified.

Results In total, 2011 patients were included in the analysis. Of these, $0.8 \%$ had hypokalemia before bowel cleansing with PEG-asc. After bowel preparation, 5.4\% developed hypokalemia. Of the patients, 281 were considered to have "high cardiac risk." The combination of "high cardiac risk" and hypokalemia was present in $1 \%$ of the initial colonoscopy population. Female sex, colorectal cancer diagnosis, and thiazide use were found to be significant predictors for hypokalemia after use of PEG-asc. No arrhythmias or serious adverse events due to hypokalemia occurred.

Conclusions Physicians referring patients for colonoscopy should be aware that "high cardiac risk" patients and those on thiazide diuretics undergoing bowel cleansing for colonoscopy are a risk of developing post-cleansing hypokalemia but it remains to be determined whether their risk of developing life-threatening arrhythmias is truly increased. 


\section{Introduction}

Worldwide, colonoscopy is extensively used for diagnostic and therapeutic indications, for surveillance, and in colorectal cancer (CRC) screening programs [1,2]. High quality and safety of colonoscopy are prerequisites for daily use in endoscopy practice. Bowel cleansing is an inseparable part of the colonoscopy procedure; therefore, high quality and safety standards for bowel cleansing have to be met.

Several bowel cleansing preparations are available, including the high-volume regimens ( $\geq 3 \mathrm{~L}$ ) polyethylene glycol (PEG) and the low-volume regimens such as sodium phosphate ( $\mathrm{NaP})$, sodium picosulphate with magnesium citrate (SPMC) and lowvolume PEG [3]. All PEG-based cleansing preparations have been developed to clean the colonic mucosal surface area without interfering with colonic electrochemical gradients or fluid absorption absorption [4] and are widely recommended [5-7]. To improve patient compliance, low-volume PEG bowel cleansing preparations have been developed. The addition of ascorbic acid to low-volume PEG (PEG-asc) has resulted in a reduction of the volume of the preparation to $2 \mathrm{~L}$. Ascorbic acid is poorly absorbed, employing an osmotic effect, synergistically with PEG $[5,8]$.

Previous studies have pointed to the risk of developing hypokalemia after PEG-based bowel cleansing preparations [7,911]. Hypokalemia may increase the risk of ventricular arrhythmia and sudden cardiac death by an imbalance of resting membrane potential difference, depolarization, and cellular hyperpolarity. Mostly, hypokalemia remains asymptomatic because alterations in serum potassium are mild $[3,12,13]$. However, more pronounced alterations in serum potassium can lead to symptoms such as fatigue, muscle weakness, constipation, and also life-threatening impaired respiration and cardiac arrhythmias [3, 12].

Previously, we reported on two fatal cases of severe hypokalemia after bowel cleansing with low-volume PEG-asc for colonoscopy [9]. These events challenged us to investigate the prevalence of hypokalemia by measuring serum potassium levels in the setting before and after bowel cleansing in high-risk groups of patients, namely diuretic users and hospitalized patients. We showed that $23.6 \%$ of the "high-risk" patients developed hypokalemia after bowel preparation [10]. It should be noted that this study has been performed in preselected groups of diuretic users and hospitalized patients. Therefore, a detailed risk factor analysis for hypokalemia could not be provided. This prompted us to perform a large, prospective study in non-selected patients scheduled for colonoscopy in regular care and screening practice. In this non-selected study population, our primary aim was to obtain data on the prevalence of hypokalemia after bowel cleansing with PEG-asc and to identify patients at significant risk of developing hypokalemia.

\section{Patients and methods}

From January 1 to July 31, 2016, we prospectively measured serum potassium, sodium, magnesium concentrations, and kidney function (serum creatinine) in all patients who underwent

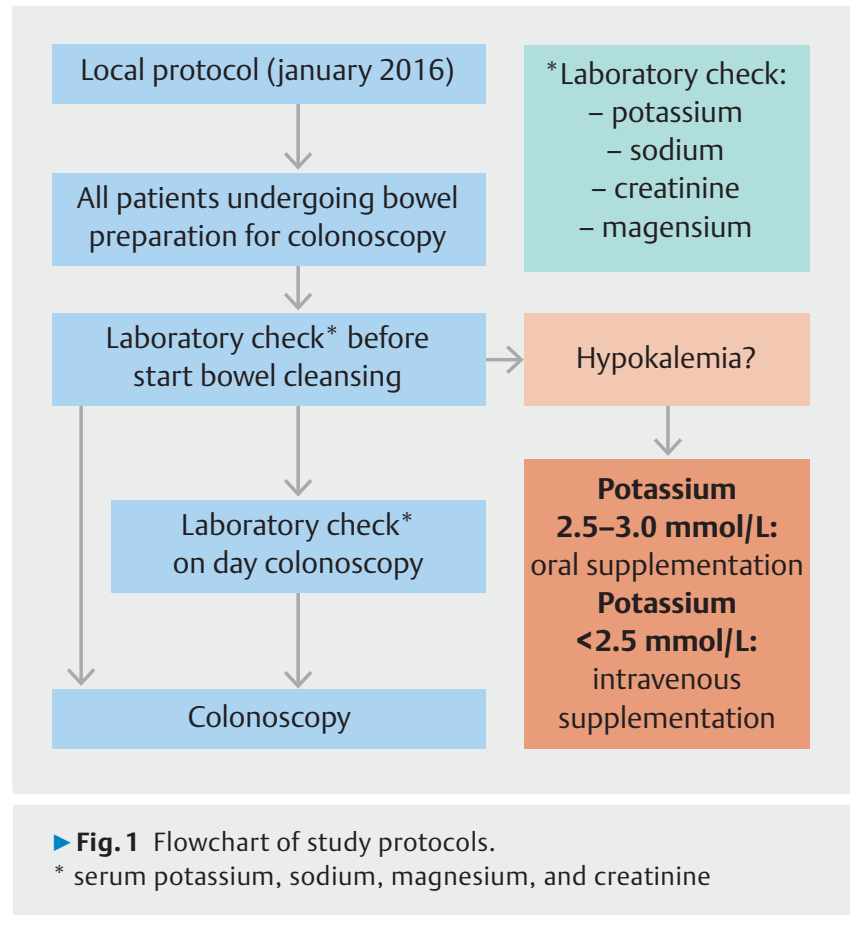

colonoscopy at our institution before and after bowel cleansing with low-volume PEG-asc bowel cleansing preparation ( $>$ Fig. 1). Magnesium values were measured given the fact that preexisting hypomagnesemia could lead to hypokalemia [14]. Clinical information, including demographic features, comorbidities, medication, and data from colonoscopy, were retrieved from digital colonoscopy reports and medical charts. All individuals received at least $2 \mathrm{~L}$ of PEG-asc as bowel cleansing preparation.

Hypokalemia is generally defined as a serum potassium value $<3.5 \mathrm{mmol} / \mathrm{L}$ [13], corresponding with the reference values in the hospital where our study was performed. Severe hypokalemia is defined as potassium values $<2.5 \mathrm{mmol} / \mathrm{L}$, moderate hypokalemia as $2.5-2.9 \mathrm{mmol} / \mathrm{L}$, and mild hypokalemia as 3.0 to $<3.5 \mathrm{mmol} / \mathrm{L}$. The normal range of serum potassium levels at our institution is between 3.5 and $5.0 \mathrm{mmol} / \mathrm{L}$. Patients diagnosed with hypokalemia before bowel cleansing were given potassium supplementation. Oral potassium supplementation (3 doses per day of $30 \mathrm{mmol}$ potassium chloride for 5 days) was provided in case of mild or moderate hypokalemia. Intravenous supplementation was provided in case of severe hypokalemia, according to our local protocol ( $\mathbf{F i g} \mathbf{1}$ ). The patients with hypokalemia at T0 were not included in the secondary analysis. The bowel cleansing preparation was given according to protocol and colonoscopy was performed. Serum potassium levels after the bowel cleansing were sampled and measured on the day the colonoscopy was performed but were not reported to the endoscopists because they were obtained for research purposes.

In regular care, monitoring of serum potassium values is considered appropriate in patient populations vulnerable to cardiac arrhythmias, such as those with a history of myocardial infarction, ischemic heart disease, heart failure or those taking 


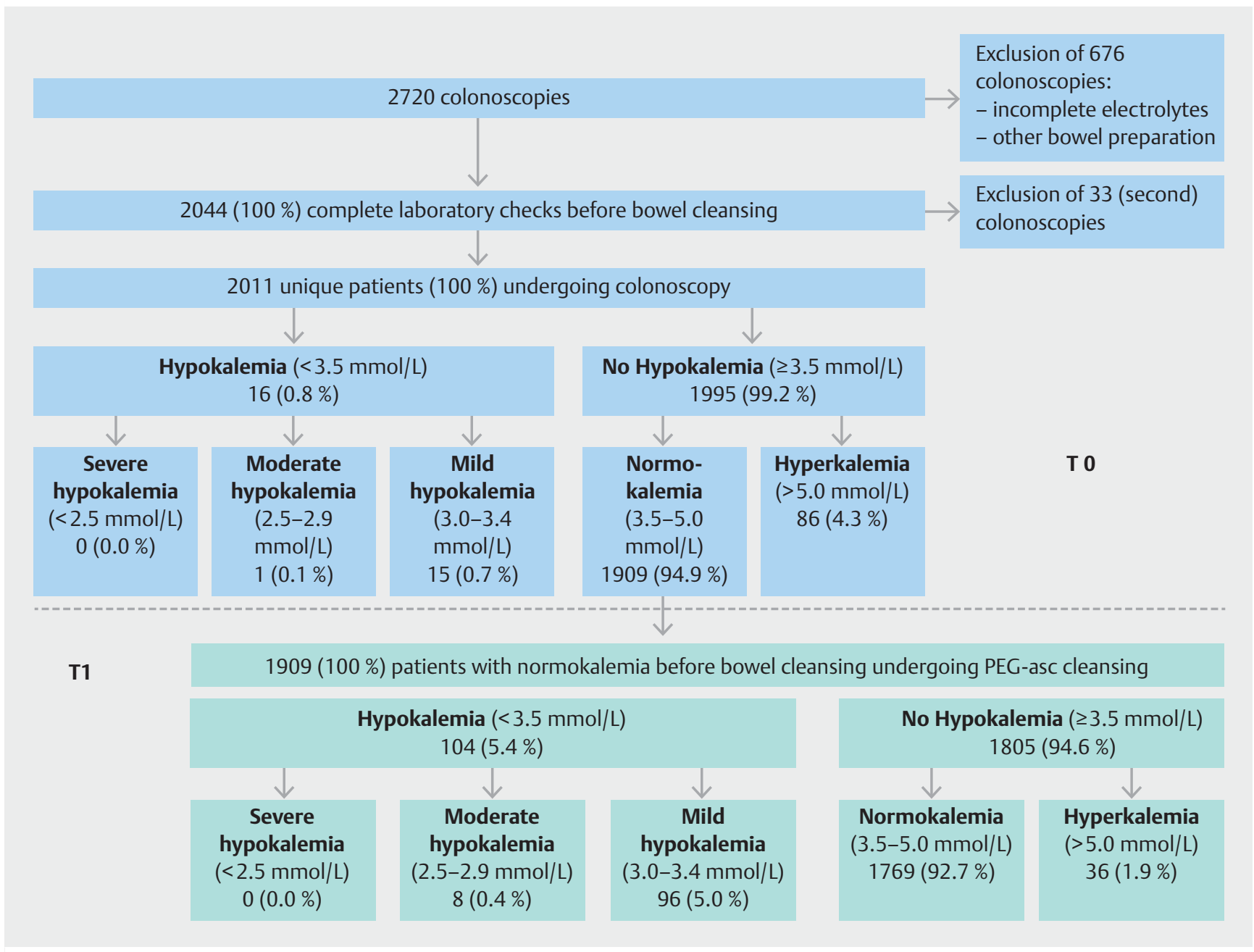

Fig. 2 Flowchart of results of T0 (patients screened before bowel cleansing) and T1 (patients screened after bowel cleansing).

digoxin. These subgroups of patients have been included as a separate "high cardiac risk" group in our analysis. This study was approved by the Institutional Review Board of our hospital and registered in The Netherlands Trial Registry: NTR5744 (http://www.trialregister.nl).

\section{Outcome measures and statistical analysis}

Statistical analyses were conducted using IBM SPSS Statistics for Windows version 26 (Armonk, New York, United States).

The primary outcome was to determine the prevalence of hypokalemia in patients after low-volume PEG-asc bowel cleansing preparation (T1) and to identify significant risk factors for developing hypokalemia. The secondary outcome was to determine the prevalence of hypokalemia before bowel preparation (T0). The patients who already had hypokalemia before bowel cleansing were excluded from analysis at T1 because: 1 ) actions to correct hypokalemia were undertaken between $\mathrm{T} 0$ and $\mathrm{T} 1$; and 2 ) it was not clear whether the risk factors identified at T1 were also present at T0. In addition to potassium, also sodium, magnesium and creatinine levels were measured.
Numerical variables are expressed as mean \pm standard deviation (SD), whereas categorical variables are presented as absolute values with percentages. Absolute values in serum electrolyte parameters were compared between T0 and T1 using paired-sample t-tests. Differences between groups (hypokalemia versus normokalemia) were analyzed using the chi-square test or Fisher's exact test where appropriate for categorical variables and the independent-samples t-test for numerical variables. Multiple logistic regression analysis was used to study whether age, sex, American Society of Anesthesiologists classification, medical history of hypertension, diuretic use (thiazide diuretics), $\beta$-blockers use, use of proton pump inhibitors, hypomagnesemia at T0, CRC diagnosed at colonoscopy, and setting (outpatient vs hospitalized) were independent risk factors for hypokalemia (versus normokalemia; excluding hyperkalemia). All variables with $P \leq 0.05$ in the univariable analysis were included in the multivariable analysis. All odds ratios (OR) are presented with $95 \%$ confidence intervals $(\mathrm{CI})$. Two-sided $P \leq$ 0.05 was considered statistically significant. 
- Table 1 Comparison of proportional changes in serum electrolytes before and after bowel preparation with PEG-asc

\begin{tabular}{|l|l|l|l|l|l|l|}
\hline & N & $\begin{array}{l}\text { Value before intake } \\
\text { (mean } \pm \text { SD, range) }\end{array}$ & N & $\begin{array}{l}\text { Value after intake } \\
\text { (mean } \pm \text { SD, range) }\end{array}$ & $\begin{array}{l}\text { Change } \\
\text { (mean } \pm \text { SD) }\end{array}$ \\
\hline Potassium & 1873 & $4.3 \pm 0.3(3.5-5.0)$ & 1873 & $4.0 \pm 0.4(2.6-5.0)$ & 1824 & $-0.3 \pm 0.4$ \\
\hline Sodium & 1828 & $140.6 \pm 2.4(128-154)$ & 1868 & $139.8 \pm 2.9(111-149)$ & 1823 & $-0.7 \pm 4.3$ \\
\hline Creatinine & 1877 & $85.1 \pm 29(11-533)$ & 1889 & $79.8 \pm 25(23-413)$ & 1872 & $-5.3 \pm 10.7$ \\
\hline Magnesium & 1564 & $0.85 \pm 0.1(0.26-1.14)$ & 1818 & $0.81 \pm 0.08(0.28-1.15)$ & 1547 & $-0.04 \pm 0.08$ \\
\hline
\end{tabular}

\section{Results}

Between January 1 and July 31, 2016, 2720 colonoscopies were performed in our center. We excluded 676 colonoscopies because of incomplete electrolyte measurements or use of bowel-preparations other than PEG-asc bowel cleansing. Of the remaining 2044 colonoscopies, 33 were second colonoscopies within an interval of several weeks for various reasons (therapeutic intervention, technical issue, insufficient bowel cleansing) and were therefore excluded. A total of 2011 unique patients (index colonoscopies) were included in the analysis ( $\vee$ Fig. 2). At baseline (T0) 16 patients (0.8\%) were found to have hypokalemia compared to 1995 patients without hypokalemia, consisting of 1909 (94.9\%) patients with normal potassium values and 86 (4.3\%) with hyperkalemia.

The mean \pm SD time from T0 until colonoscopy was $12.0 \pm$ 15.8 days, range $0-117$ days. The sixteen patients with hypokalemia at $\mathrm{T} 0$ received potassium supplementation according to protocol, as previously described. Of these sixteen patients, six patients (37.5\%) had hypokalemia on the day of colonoscopy, despite potassium supplementation (mean \pm SD of potassium at T1: $3.3 \pm 0.5 \mathrm{mmol}$, range $2.4-4.3 \mathrm{mmol} / \mathrm{L}$ ).

After bowel preparation, 104 of 1909 patients (5.4\%) had developed hypokalemia on the day of colonoscopy (T1) ( $\mathbf{F i g .}$ 2 ), including eight $(0.4 \%)$ with moderate hypokalemia and 96 (5.0\%) with mild hypokalemia but no patient had severe hypokalemia. The other 1805 patients (94.6\%) did not develop hypokalemia: 1769 (92.7\%) with normokalemia and 36 (1.9\%) with hyperkalemia. As a group, potassium levels at T1 declined on average with $0.3( \pm 0.4) \mathrm{mmol} / \mathrm{L}$ compared to potassium levels at T0 ( $>$ Table 1$)$. Not only serum potassium levels but also sodium, magnesium and creatinine levels at T1 were significantly lower compare to T0 ( $\triangleright$ Table $\mathbf{1}$ ).

The multivariable logistic regression model ( $\triangleright$ Table 2 ) showed that hypokalemia was significantly more frequent in the group of thiazide diuretic users compared to non-users (adjusted OR 3.64, 95\% Cl 1.97-6.70, $P<0.001$ ), in the group diagnosed with CRC versus no CRC at colonoscopy (adjusted OR $4.42,95 \% \mathrm{Cl} 1.85-10.53, P=0.001)$ and in the group with female versus male sex (adjusted OR 1.71, 95\% Cl 1.04-2.81, $P=$ $0.035)$. There was no statistically significant difference in developing hypokalemia at T1 between the single-drug thiazide diuretic users and the combination of thiazide diuretics with angiotensin-converting enzyme (ACE) or angiotensin-II inhibitor users $(\mathrm{N}=19)$ (single use vs combination, OR $1.97,95 \% \mathrm{Cl}$
0.93-4.18, $P=0.08$ ) (data not shown in $>$ Table 2). While in general hypokalemia can result from hypomagnesemia, multiple logistic regression did not show that hypomagnesemia was significantly more frequent in the hypokalemia group compared to the normokalemia group. Of the 40 patients with hypomagnesemia, $23(57.5 \%)$ were on permanent use of proton pump inhibitors (PPI).

As shown in $>$ Table 2 and $>$ Table 3, 281 patients belonged to the "high cardiac risk" group. Of them, 20 patients developed hypokalemia after bowel cleansing. This group with a "high cardiac risk" combined with hypokalemia consists of $1 \%$ (20/1909) of the initial colonoscopy population and is considered to be most prone to develop cardiac arrhythmias. No cardiac arrhythmias or other adverse events (AEs) occurred in our study population.

\section{Discussion}

This real-life evaluation in regular endoscopy practice showed that the prevalence of hypokalemia in the non-selected group of patients referred for colonoscopy was: 1 ) $0.8 \%$ in patients before start of bowel cleansing for colonoscopy (T0); and 2) $5.4 \%$ in patients after bowel cleansing with low-volume PEGasc (T1) who had normal potassium levels before bowel cleansing. In our non-selected group, the prevalence of post-cleansing hypokalemia appeared to be low with only cases of mild $(5.0 \%)$ and moderate $(0.4 \%)$ hypokalemia, without any case of severe hypokalemia.

With respect to risk factors for hypokalemia: Patients developed hypokalemia after bowel cleansing significantly more frequent if they were female, had a diagnosis of CRC confirmed during colonoscopy, and/or were using a thiazide diuretic. Female sex has previously been described as a predictor of hypokalemia, possibly related to lower lean body mass compared to male sex $[15,16]$. In general, up to $40 \%$ of all patients treated with thiazide diuretics develop hypokalemia $[13,17]$. Thus, hypokalemia is a regularly occurring consequence of use of thiazide diuretics [18]. CRC diagnosis appeared to be a third risk factor in our analysis. Hypokalemia is very common in cancer patients and is multifactorial in origin, resulting from reduced intake (cancer cachexia), increased losses (vomiting, diarrhea, renal loss), and redistribution of potassium into the intracellular compartment of tumor cells [19].

Hypokalemia is one of the most frequently encountered electrolyte abnormalities in daily clinical practice: up to $20 \%$ of 
- Table 2 Clinical characteristics of subjects with hypokalemia versus normokalemia after bowel cleansing - T1.

\begin{tabular}{|c|c|c|c|c|c|c|}
\hline & \multirow{2}{*}{$\begin{array}{l}\text { Hypokalemia } \\
\mathrm{N}=104\end{array}$} & \multirow{2}{*}{$\begin{array}{l}\text { Normokalemia } \\
N=1769\end{array}$} & \multicolumn{2}{|l|}{ Unadjusted } & \multicolumn{2}{|l|}{ Adjusted } \\
\hline & & & OR $(95 \% \mathrm{Cl})$ & $P$ value & OR (95\% CI) & $P$ value \\
\hline Age, yrs (mean $\pm S D$ ) & $65.6 \pm 13.5$ & $61.1 \pm 14.6$ & $1.02(1.01-1.04)$ & $0.003^{1}$ & $0.99(0.98-1.02)$ & 0.81 \\
\hline Sex, female & $64(61.5 \%)$ & $826(46.7 \%)$ & $1.84(1.23-2.76)$ & $0.003^{1}$ & $1.71(1.04-2.81)$ & $0.035^{1}$ \\
\hline ASA & & & & $0.03^{1}$ & & 0.45 \\
\hline 1 & $25(24.0 \%)$ & $585(33.1 \%)$ & Ref & & Ref & \\
\hline 2 & $70(67.3 \%)$ & $1110(67.3 \%)$ & $1.48(0.93-2.35)$ & 0.10 & $1.07(0.58-1.99)$ & 0.82 \\
\hline 3 & $9(8.7 \%)$ & $74(4.2 \%)$ & $2.85(1.28-6.33)$ & $0.01^{1}$ & $1.99(0.65-6.07)$ & 0.23 \\
\hline Setting, hospitalized & $8(7.7 \%)$ & $55(3.1 \%)$ & $2.60(1.20-5.61)$ & $0.015^{1}$ & $1.22(0.24-6.14)$ & 0.81 \\
\hline Indication & & & & 0.91 & & \\
\hline Symptoms & $59(56.7 \%)$ & $1005(56.8 \%)$ & Ref & & & \\
\hline Screening & $21(20.2 \%)$ & $311(17.6 \%)$ & $1.15(0.69-1.92)$ & 0.60 & & \\
\hline Surveillance & $24(23.1 \%)$ & $446(25.2 \%)$ & $0.92(0.56-1.49)$ & 0.73 & & \\
\hline Therapeutic & $0(0 \%)$ & $7(0.4 \%)$ & 2 & 1.00 & & \\
\hline \multicolumn{7}{|l|}{ Indication, symptoms } \\
\hline Stool change & $13(12.5 \%)$ & $239(13.5 \%)$ & $0.92(0.50-1.66)$ & 0.77 & & \\
\hline Anemia & $3(2.9 \%)$ & $39(2.2 \%)$ & 2 & 0.65 & & \\
\hline Hematochezia & $22(21.2 \%)$ & $397(22.4 \%)$ & $0.93(0.57-1.50)$ & 0.76 & & \\
\hline Diarrhea & $13(12.5 \%)$ & $182(10.3 \%)$ & $1.25(0.68-2.27)$ & 0.47 & & \\
\hline Abdominal pain/discomfort & $8(7.7 \%)$ & $148(8.4 \%)$ & $0.91(0.44-1.91)$ & 0.81 & & \\
\hline \multicolumn{7}{|l|}{ Laboratory findings T0 } \\
\hline Hypomagnesemia $(N=1548)$ & $7(9.1 \%)$ & $33(2.2 \%)$ & $4.36(1.86-10.20)$ & $0.001^{1}$ & $2.32(0.86-6.25)$ & 0.097 \\
\hline$C K D, M D R D<30$ & $0(0 \%)$ & $14(0.8 \%)$ & 2 & 1.00 & & \\
\hline \multicolumn{7}{|l|}{ Colonoscopy findings } \\
\hline$B B P S \geq 6$ & $91(87.5 \%)$ & $1613(91.2 \%)$ & $0.68(0.37-1.24)$ & 0.21 & & \\
\hline Adenoma & $26(25.0 \%)$ & $517(29.2 \%)$ & $0.81(0.51-1.27)$ & 0.36 & & \\
\hline Diverticulosis & $26(4.5 \%)$ & $549(31.1 \%)$ & $0.74(0.47-1.17)$ & 0.20 & & \\
\hline CRC & $10(9.6 \%)$ & $64(3.6 \%)$ & $2.68(1.33-5.36)$ & $0.006^{1}$ & $4.42(1.85-10.53)$ & $0.001^{1}$ \\
\hline Active IBD & $98(94.2 \%)$ & $1710(96.7 \%)$ & $0.56(0.24-1.34)$ & 0.19 & & \\
\hline \multicolumn{7}{|l|}{ Medication } \\
\hline Thiazide diuretics & $34(32.7 \%)$ & $182(10.3 \%)$ & $4.28(2.76-6.63)$ & $<0.001^{1}$ & $3.64(1.97-6.70)$ & $<0.001^{1}$ \\
\hline Loop diuretics & $9(8.7 \%)$ & $80(4.5 \%)$ & $2.02(0.98-4.14)$ & 0.056 & & \\
\hline Beta-blockers & $40(38.5 \%)$ & $358(20.2 \%)$ & $2.45(1.62-3.70)$ & $<0.001^{1}$ & $1.27(0.70-2.30)$ & 0.43 \\
\hline RAAS activators & $34(32.7 \%)$ & $452(25.6 \%)$ & $1.40(0.92-2.14)$ & 0.12 & & \\
\hline Protonpumpinhibitors & $40(38.5 \%)$ & $507(28.7 \%)$ & $1.56(1.03-2.34)$ & $0.034^{1}$ & $1.30(0.77-2.19)$ & 0.33 \\
\hline \multicolumn{7}{|l|}{ Medical history } \\
\hline IBD & $4(3.8 \%)$ & $48(2.7 \%)$ & 2 & 0.50 & & \\
\hline CRC & $8(7.7 \%)$ & $115(6.5 \%)$ & $1.20(0.57-2.53)$ & 0.63 & & \\
\hline Hypertension & $56(53.8 \%)$ & $610(34.5 \%)$ & $2.22(1.49-3.30)$ & $<0.001^{1}$ & $1.35(0.70-2.61)$ & 0.37 \\
\hline Diabetes mellitus & $11(10.6 \%)$ & $196(11.1 \%)$ & $0.95(0.50-1.80)$ & 0.87 & & \\
\hline High cardiac risk ${ }^{3}$ & $20(19.2 \%)$ & $261(14.8 \%)$ & $1.38(0.83-2.28)$ & 0.22 & & \\
\hline
\end{tabular}


- Table 2 (Continuation)

ASA, American Society of Anesthesiologists; CKD, chronic kidney disease; MDRD, modification of diet in renal disease; BBPS, Boston Bowel Preparation Scale; CRC, colorectal cancer; IBD, irritable bowel disease; RAAS, renin-angiotensin-aldosterone system.

$1 P<0.05$

${ }^{2}$ Fisher's exact test used.

3 Patients vulnerable to cardiac arrhytmias, see also $\triangleright$ Table 3.

- Table 3 High cardiac risk- at T1 hypokalemia vs normokalemia after bowel cleansing.

\begin{tabular}{|c|c|c|c|c|}
\hline & \multirow{2}{*}{$\begin{array}{l}\text { Hypokalemia } \\
\mathrm{N}=104\end{array}$} & \multirow{2}{*}{$\begin{array}{l}\text { Normokalemia } \\
\mathrm{N}=1769\end{array}$} & \multicolumn{2}{|l|}{ Unadjusted } \\
\hline & & & OR $(95 \% \mathrm{Cl})$ & $P$ value \\
\hline Congestive heart failure & $4(3.8 \%)$ & $40(2.3 \%)$ & 1 & 0.31 \\
\hline Ischemic heart disease & $10(9.6 \%)$ & $142(8.0 \%)$ & $1.22(0.62-2.39)$ & 0.57 \\
\hline \multicolumn{5}{|l|}{ Serious arrhythmias } \\
\hline Atrial tachycardia ${ }^{1}$ & $10(9.6 \%)$ & $128(7.2 \%)$ & $1.36(0.69-2.68)$ & 0.37 \\
\hline Ventricular tachycardia ${ }^{2}$ & 0 & $1(0.6 \%)$ & - & 1.00 \\
\hline Bradycardia & 0 & $2(0.1 \%)$ & - & 1.00 \\
\hline Digoxin use & 0 & $19(1.1 \%)$ & - & 1.00 \\
\hline
\end{tabular}

hospitalized patients are diagnosed with hypokalemia, but only in $4 \%$ to $5 \%$ hypokalemia becomes symptomatic with symptoms ranging from mild and moderate (muscle weakness, nausea, vomiting, constipation) to severe (seizures, paralysis, arrhythmias, coma and mortality) [15, 20-22].

It is well documented that patients with congestive heart failure, ischemia, or a history of arrhythmias have a much higher risk of developing life-threatening arrhythmias when they are hypokalemic [13]. Of the initial colonoscopy population, 281 patients (15\%) belonged to the "cardiac high risk" group. Of these, 20 patients $(7.1 \%)$ developed hypokalemia after bowel cleansing ( Table 2 and $>$ Table 3 ) and $40 \%$ of these 20 patients used thiazide diuretics (OR $3.4895 \% \mathrm{Cl} 1.34-9.02$ ), $P=$ 0.010 ). This group with high cardiac risk and hypokalemia is considered to have an additive, significantly higher risk of developing cardiac arrhythmias but is very small and consists of only $1 \%$ of the initial colonoscopy population.

In general, potassium monitoring is considered appropriate in populations that are more prone to develop cardiac arrhythmias, such as patients with a history of ischemic heart disease or myocardial infarction, heart failure or those taking digoxin and in patients at risk of developing hypokalemia.

Magnesium is an important cofactor to maintain intracellular potassium levels within normal limits. In particular magnesium depletion results in secondary potassium depletion [23]. In our study, no significant association was found between hypokalemia and PPI use or hypomagnesemia ( $>$ Table 2 ). In general, screening for hypomagnesemia in chronic PPI users is not recommended [24]. However, hypomagnesemia should be considered in case of clinically relevant hypokalemia, because prevalences up to $50 \%$ have been reported [21].

In daily endoscopy practice, data on cardiac arrhythmia events and electrolyte disturbances are lacking. These missing data are necessary before any recommendation on screening for hypokalemia in endoscopy practice can be made. Therefore, complications due to bowel cleansing, such as electrolyte disturbances and cardiac rhythm disturbances, should be more systematically reported.

A strength of our analysis is the complete capture of all patients undergoing colonoscopy during a specific time interval. The current study is the first prospective, population-based and unselected study reporting prevalences of hypokalemia before and after PEG-asc preparation in a regular colonoscopy population.

Some limitations also have to be taken into account. First, we did not systematically screen for symptoms of hypokalemia in our study. Second, in patients with hypokalemia at T0, shortterm supplementation of potassium was provided but further management was handed over to their general practitioner. A significant proportion (37.5\%) of these patients, again, appeared to have hypokalemia at T1. None of these patients had a "high cardiac risk" profile. In case hypokalemia is diagnosed and treatment is initiated, follow up with potassium measurement after supplementation is indicated before further actions such as bowel cleansing and colonoscopy are undertaken. Third, due to the moderate number of 104 cases with hypokalemia out of a total of 1909 cases, only the variables with $P<$ 0.05 in the univariable analysis were included in the multivariable analysis. 
From a practical clinical point of view:, the data generated in this study do not provide sufficient evidence for additional screening for hypokalemia in patients undergoing bowel cleansing and colonoscopy apart from general recommendations. However, special awareness is needed in the group of patients with hypokalemia prior to bowel preparation $(0.8 \%)$, because they could be more prone to develop life-threatening arrhythmias. In our study, this group received potassium supplementation and this may have influenced the zero AEs.

\section{Conclusions}

In conclusion, in this large colonoscopy population-based study we have shown that hypokalemia was present in: 1) $0.8 \%$ of patients before bowel cleansing with PEG-asc and; 2) in $5.4 \%$ of patients after completing bowel cleansing with PEG-asc who had normal potassium values before bowel cleansing. Female sex, CRC diagnosis, and thiazide use were found to be significant predictors of hypokalemia after use of PEG-asc. Physicians referring patients for colonoscopy should be aware that "high cardiac risk" patients and those on thiazide diuretics undergoing bowel cleansing for colonoscopy are at risk of developing post-cleansing hypokalemia but it remains to be determined whether their risk of developing life-threatening arrhythmias is truly increased.

\section{Acknowledgements}

The authors thank Silvia Sanduleanu, MD, PhD, GROW, School for Oncology and Developmental Biology, University of Maastricht, Maastricht, The Netherlands, for help on the study protocol.

\section{Competing interests}

The authors declare that they have no conflict of interest.

\section{References}

[1] Rex DK, Schoenfeld PS, Cohen J et al. Quality indicators for colonoscopy. Gastrointest Endosc 2015; 81: 31-53

[2] Stock C, Haug U, Brenner H. Population-based prevalence estimates of history of colonoscopy or sigmoidoscopy: review and analysis of recent trends. Gastrointest Endosc 2010; 71: 366-381.e362

[3] Tan J], Tjandra JJ. Which is the optimal bowel preparation for colonoscopy - a meta-analysis. Colorectal Dis 2006; 8: 247-258

[4] Fordtran JS, Hofmann AF. Seventy years of polyethylene glycols in gastroenterology: the journey of PEG 4000 and 3350 from nonabsorbable marker to colonoscopy preparation to osmotic laxative. Gastroenterology 2017; 152: 675-680
[5] Johnson DA, Barkun AN, Cohen LB et al. Optimizing adequacy of bowel cleansing for colonoscopy: recommendations from the US multi-society task force on colorectal cancer. Gastroenterology 2014; 147: 903-924

[6] Hassan C, East J, Radaelli F et al. Bowel preparation for colonoscopy: European Society of Gastrointestinal Endoscopy (ESGE) Guideline Update 2019. Endoscopy 2019; 51: 775-794

[7] Saltzman JR, Cash BD, Pasha SF et al. Bowel preparation before colonoscopy. Gastrointest Endosc 2015; 81: 781-794

[8] Fujita I, Akagi Y, Hirano J et al. Distinct mechanisms of transport of ascorbic acid and dehydroascorbic acid in intestinal epithelial cells (IEC-6). Res Commun Mol Pathol Pharmacol 2000; 107: 219-231

[9] Reumkens A, Masclee AAM, Bakker CM. Postcolonoscopy mortality: Bowel preparation to blame? Gastrointest Endosc 2017; 86: 744-745

[10] Reumkens A, Masclee AA, Winkens B et al. Prevalence of hypokalemia before and after bowel preparation for colonoscopy in high-risk patients. Gastrointest Endosc 2017; 86: 673-679

[11] Ho JM, Juurlink DN, Cavalcanti RB. Hypokalemia following polyethylene glycol-based bowel preparation for colonoscopy in older hospitalized patients with significant comorbidities. Ann Pharmacother 2010; 44: 466-470

[12] Hoorn EJ, Tuut MK, Hoorntje SJ et al. Dutch guideline for the management of electrolyte disorders - 2012 revision. Netherlands J Med 2013; 71: 153-165

[13] Cohn JN, Kowey PR, Whelton PK et al. New guidelines for potassium replacement in clinical practice: a contemporary review by the $\mathrm{Na}-$ tional Council on Potassium in Clinical Practice. Arch Int Med 2000; 160: 2429-2436

[14] Ayuk J, Gittoes NJ. How should hypomagnesaemia be investigated and treated? Clin Endocrinol 2011; 75: 743-746

[15] Nilsson E, Gasparini A, Arnlov J et al. Incidence and determinants of hyperkalemia and hypokalemia in a large healthcare system. Int J Cardiol 2017; 245: 277-284

[16] Kleinfeld M, Borra S, Gavani S et al. Hypokalemia: are elderly females more vulnerable? J Natl Med Assoc 1993; 85: 861-864

[17] Gennari FJ. Hypokalemia. N Engl J Med 1998; 339: 451-458

[18] Ellison DH, Loffing J. Thiazide effects and adverse effects: insights from molecular genetics. Hypertension 2009; 54: 196-202

[19] Liamis G, Filippatos TD, Elisaf MS. Electrolyte disorders associated with the use of anticancer drugs. Euro J Pharmacol 2016; 777: 78-87

[20] Pepin J, Shields C. Advances in diagnosis and management of hypokalemic and hyperkalemic emergencies. Emerg Med Pract 2012; 14: 1-17; quiz $17-18$

[21] Udensi UK, Tchounwou PB. Potassium homeostasis, oxidative stress, and human disease. Int J Clin Exp Physiol 2017; 4: 111-122

[22] Clayton JA, Rodgers S, Blakey J et al. Thiazide diuretic prescription and electrolyte abnormalities in primary care. Br J Clin Pharmacol 2006; 61: 87-95

[23] Whang R, Whang DD, Ryan MP. Refractory potassium repletion. A consequence of magnesium deficiency. Arch Int Med 1992; 152: 40-45

[24] Freedberg DE, Kim LS, Yang YX. The Risks and benefits of long-term use of proton pump inhibitors: expert review and best practice advice from the American Gastroenterological Association. Gastroenterology $2017 ; 152: 706-715$ 